\title{
THICKNESS IN TOPOLOGICAL TRANSFORMATION SEMIGROUPS
}

\section{TYLER HAYNES}

\author{
Mathematics Department \\ Saginaw Valley State University \\ University Center, Michigan 48710-0001 \\ (Received June 27, 1989 and in revised form February 5, 1993)
}

\begin{abstract}
This article deals with thickness in topological transformation semigroups ( $\tau$-semigroups). Thickness is used to establish conditions guaranteeing an invariant mean on a function space defined on a $\tau$-semigroup if there exists an invariant mean on its functions restricted to a sub- $\tau$-semigroup of the original $\tau$-semigroup. We sketch earlier results, then give many cquivalent conditions for thickness on $\tau$-semigroups, and finally present theorems giving conditions for an irvariant mean to exist on a function space.
\end{abstract}

KEY WORDS AND PHRASES. Thickness, topological transformation semigroup. transformation semigroup, invariant mean

1980 AMS SUBJECT CLASSIFICATION CODES. 22A20, 43A07

\section{Left-Thickness in Semigroups}

Mitchell introduced the concept of left-thickness in a semigroup [Mitchell, 1965]: a subset $T$ of semigroup $S$ is left-thick in $S-\forall$ finite $U \subseteq S, \exists t e S: U t=T$.

Any left ideal of a semigroup is left-thick, but not conversely. The complete relationship between left ideals and left-thick subsets is this: $\operatorname{Let} \beta(S)$ be the Stonc-Cech compactification of semigroup $S$ endowed with the discrete topology, and let $T \subseteq S$. Then $T$ is left-thick in $S-$ the closure of $T$ in $\beta(S)$ contains a left ideal of $\beta(S)$ [Wilde \& Witz. 1967. lemma 5.1]. (See Theorem 4.3.g infra for a more general formulation of this result.)

It can be shown that in the definition $t$ can be taken in $T$ or $U$ can be a singleton.

Let $B(S)=$ the set of all bounded complex- or real-valued functions on semigroup $S$. For any $s \in S$ and $f \in B(S), T_{s} f$ denotes the function in $B(S)$ defined by $T_{s} f(t)=f(s t)$ ( $\left.\forall t \in S\right)$.

$A$ mean on $B(S)$ is a member of the dual space $B(S)^{*}$ of $B(S)$ which satisfies $\mu(1)=1=$ |p|. Mean $\mu$ is invariant $\rightarrow \mu\left(\mathrm{T}_{s} \mathrm{f}\right)=\mu \mathrm{f}(\forall s \in \mathrm{S}, \mathrm{f} \in \mathrm{B}(\mathrm{S}))$.

The importance of left-thickness for our subject is because of this theorem [Mitchell, 1965, theorem 9].

Theorem. Let $T$ be a left-thick subsemigroup of semigroup $S$. Then $B(S)$ has a leftinvariant mean $-B(T)$ has a left-invariant mean.

H. D. Junghenn generalized Mitchell's concept of left-thickness [Junghenn, 1979, p. 38]. First it is necessary to define more terms.

Subspace $F$ of $B(S)$ is left-translation invariant $-T_{s} f \in F(\forall s \in S, f \in F)$. Let $\mu \in F^{*}$, the dual space of $F$; define $T_{\mu} f(\forall f \in F)$ by $T_{\mu} f(s)=\mu\left(T_{s} f\right)(\forall s \in S)$. Then $T_{\mu}: F \rightarrow B(S)$. F is left-introverted $T_{\mu}(F) \subseteq F\left(\forall \mu \epsilon F^{*}\right)$.

Definition. Let $S$ be a semigroup; $F \subseteq B(S)$ be a left-translation invariant, left-introverted. norm-closed subalgebra containing the constant functions: $T \subseteq S$ be non-empty; $\mathrm{F}(\mathrm{T})=\left\{\mathrm{g} \in \mathrm{F} \mid \chi_{\mathrm{T}} \leq \mathrm{g} \leq 1\right\}$. Then

$\mathrm{T}$ is $F$-left thick in $\mathrm{S}-\forall \epsilon>0, \mathrm{~g} \in \mathrm{F}(\mathrm{T})$, and finite $\mathrm{U}=\left\{\mathrm{s}_{1}, \mathrm{~s}_{2}, \ldots, \mathrm{s}_{\mathrm{n}}\right\} \in \mathrm{S} \exists \mathrm{s \in S}: \mathrm{g}\left(\mathrm{s}_{1}, \mathrm{~s}\right)>1-\epsilon(\mathrm{i}=1, \ldots . \mathrm{n})$

If $\chi_{T} \in F$, then Junghenn's definition of F-left thickness reduces to Mitchell's definition of leftthickness: let $g=\chi_{T}$, then for $0<\epsilon<1,1-\epsilon<g\left(s_{1} s\right)=\chi_{T}\left(s_{1} s\right)-s_{1} s \in T(i=1, \ldots, n)$. 
Junghenn generalizes Mitchell's theorem thus:

Theorem. If $T$ is a left-thick subsemigroup of $S$, then $F$ has a left-invariant mean $\left.F\right|_{T}$ has a left-invariant mean.

\section{Transformation Semigoups}

Thickness can be defined in the more general setting of a transformation semigroup. This section defines such semigroups and other necessary terms.

Definition 21. A transformation semigroup is a system $\langle S, X, \pi\rangle$ consisting of a semigroup $S$, a sct $X$. and a mapping $\pi: S \times X \rightarrow X$ which satisfies

1. $\pi(s, \pi(t, x))=\pi(s t, x)(\forall s, t \in S, x \in X) ;$

2. $\pi(e, x)=x(\forall x \in X)$ whenever $S$ has two-sided identity $e$.

If $\pi(s, x)=s x$ expresses the image of $(s, x)$ under $\pi$, then condition (1) becomes $s(t x)=(s t) x$ and condition (2) becomes $\mathrm{ex}=\mathrm{x}$.

The abbreviated notion $\langle\mathrm{S}, \mathrm{X}\rangle$ will denote a transformation semigroup whenever the meaning of $\pi$ is clear or whenever $\pi$ is generic.

$\langle T . Y\rangle$ is a subtransformation semigroup of $\langle S . X\rangle-T$ is a subsemigroup of $S . Y \leq X$. and TY $\subseteq Y$.

Definition 22 Let scmigroup $S$ and set $X$ both be endowed with Hausdorff topologies. Transformation semigroup $\langle\mathrm{S}, \mathrm{X}, \boldsymbol{\pi}\rangle$ is a topological transformation semigroup, or $\tau$-semigroup $\pi$ is separately continuous in the variables $s$ and $x$.

Again, a $\tau$-semigroup will be denoted briefly by $\langle S, X\rangle$.

Let $C(X)$ denote the set of continuous and bounded complex- or real-valued functions on $X$.

Definition 23. Let $\langle S, X\rangle$ be a $\tau$-semigroup. $T_{s} f$ denotes, for any $s \in S$ and $f \in C(X)$, the function in $C(X)$ defined by $T_{s} f(x)=f(s x)(\forall x \in X)$. If $F$ is a linear subspace of $C(X)$, then $F$ is $S$ invariant $-T_{s} f \in F(\forall s \in S, f \in F)$. Notation: $T_{S}=\left\{T_{s} \mid s \in S\right\}$ and $T_{s} F=\left\{T_{s} f \mid f \in F\right\}$.

Observe that $T_{t} T_{s}=T_{s t}(\forall s, t \in S)$.

Definition 24. Let $\langle S, X\rangle$ be a $\tau$-semigroup: $F$ be a linear space $c C(X)$ which is normclosed, conjugate-closed. S-invariant, and contains the constant functions; $\mathrm{G} \subseteq \mathrm{C}(\mathrm{S})$ a linear space. and let $\mu \in F^{*}$. Define $T_{\mu} f(\forall \in F)$ by $T_{\mu} f(s)=\mu\left(T_{s} f\right)(\forall s \in S)$. Then $T_{\mu}: F \rightarrow B(S)$. $F$ is $G$ introverted $-\mathrm{T}_{\mu}(\mathrm{F}) \mathrm{G}\left(\forall_{\mu \in \mathrm{F}^{*}}\right)$.

In the preceding definition $\mathrm{F}^{*}$ may be replaced by $\mathrm{C}(\mathrm{X})^{*}$ since every functional in $\mathrm{F}^{*}$ can be extended to a functional in $\mathrm{C}(\mathrm{X})^{*}$. Also it can be shown that $\mathrm{F}^{*}$ can be replaced by $\mathrm{M}(\mathrm{F})$, the set of all means on $F$.

Definition 2.5. Let $F$ be $G$-introverted. $\mu \in F^{*}$, and $\lambda \epsilon G^{*}$. The evolution product of $\lambda$ and $\mu$, denoted $\lambda_{\mu}$, is defined by $\lambda_{\mu} f=\lambda\left(T_{\mu} f\right)(\forall \in F)$.

Note that $\lambda_{\mu \epsilon F^{*}}$ and that if $G$ is norm-closed, conjugate-closed, and contains the constant functions, then $\lambda \in M(G)$ and $\mu \in M(F)$ imply $\lambda \mu \in M(F)$.

A mean on $\mathrm{F} \subset \mathrm{C}(\mathrm{X})$ is defined in the same way as a mean on $\mathrm{B}(\mathrm{S})$ was defined in section 1. If $F$ is an algebra under pointwise multiplication, then mean $\mu$ is multiplicative $-\mu(f g)=\mu(f) \mu(g)$ $(\forall, g \in F)$.

Let $M(F)=$ set of all means on $F$, and $M M(F)=$ set of all multiplicative means on $F$. $M(F)$ and $M M(F)$ are both $w^{*}$-compact. being closed subsets of the unit ball in $F^{*}$.

Mean $\mu \in M(F)$ is invariant $-\mu\left(T_{s} f\right)=\mu(f)(\forall f \in F . s e S)$. Note that $\mu$ is invariant $-e(s) T_{\mu}=$ $T_{\mu}(\forall s \in S)$.

An evaluation at $x \in X$ is defined by $e(x) f=f(x)(\forall f \in F)$; clearly an evaluation is a mean. A finite mean on $\mathrm{F}$ is a convex combination of evaluations.

A mean is multiplicative if and only if it is the $w^{*}$-limit of evaluations. 
A special case of transformation semigroup is furnished by letting $X=S$ and $\pi=\lambda(\bullet)$ wherc $\lambda_{s}: S \rightarrow S$ is defined for any fixed seS by $\lambda_{s}(t)=s t(\forall t \in S)$. If $G \_C(S)$ is a linear space, then $\mathrm{L}_{\mathrm{s}} \mathrm{g}(\mathrm{t})=\mathrm{g}(\mathrm{st})(\forall s, t \in S, g \in G)$; also, $\lambda, \mu \in \mathrm{M}(\mathrm{G})-\lambda_{\mu \epsilon \mathrm{M}}(\mathrm{G})$. If $F \subset C(X)$ is a linear space then $\mathrm{L}_{s} T_{\mu}$ $=\mathrm{T}_{\mu} \mathrm{T}_{\mathrm{S}}(\forall s \in \mathrm{S}, \mu \in \mathrm{M}(\mathrm{F}))$. Mean $\mu \in \mathrm{M}(\mathrm{G})$ is left-invariant $-\mu\left(\mathrm{L}_{\mathrm{s}} \mathrm{g}\right)=\mu(\mathrm{g})(\forall \mathrm{g} \in \mathrm{G})$.

\section{Thickness in Transformation Semigroups}

Junghenn's generalization of F-left thickness carries over in a straightforward way to transformation semigroups. The corresponding concept is defined in Definition 3.1, and a plethora of alternative characterizations is given by Theorem 3.3 .

\section{Assumptions:}

$\langle\mathrm{S}, \mathrm{X}\rangle$ is a transformation semigroup;

$\mathrm{G} \subseteq \mathrm{C}(\mathrm{S})$ is a subalgebra;

$F \_C(X)$ is an algebra which is norm-closed. S-invariant, $G$-introverted, and contains the constant

functions: $\mathrm{Y} \subseteq \mathrm{X}$.

Notation:

$F(Y)=\left\{g \in F \mid \chi_{Y} \leq g \leq 1\right\}=\{g \in F \mid 0 \leq g \leq 1, g \equiv 1$ on $Y\}$

$Z(Y)=\{g \epsilon \bar{F} \mid g \equiv 0$ on $Y\}$.

Definition 3.1. $\mathrm{Y}$ is $F, S$-thick in $\mathrm{X}-\forall \epsilon>0, \mathrm{~g} \in \mathrm{F}(\mathrm{Y})$, and finite $\mathrm{U}=\left\{\mathrm{s}_{1}, \mathrm{~s}_{2}, \ldots, \mathrm{s}_{\mathrm{n}}\right\} \in S, \exists \mathrm{x} \in \mathrm{X}$ : $\mathrm{g}\left(\mathrm{s}_{\mathrm{k}} \mathrm{x}\right)>1-\epsilon(\mathrm{k}=1, \ldots, \mathrm{n})$.

Remark 3.2 If $\mathrm{X}=\mathrm{S}$ and the action is left multiplication, then the definition is identical to Junghenn's.

Relative to Theorem $3.3 \mathrm{~b}, \mathrm{~h}, \mathrm{i,j}$ infra it is necessary to recall that a norm-closed subalgebra $\mathrm{F}$ of $C(X)$ is also a closed lattice, so that, in particular, $f \in F=|f| \in F$ [Simmons, p. 159, lemma].

Theorem 3.3. The following statements are equivalent:

a. $\mathrm{Y}$ is $\mathrm{F}, \mathrm{S}$-thick in $\mathrm{X}$;

b. $\forall \epsilon>0$, finite $D=\left\{g_{1}, g_{2}, \cdots, g_{m}\right\} \subseteq F(Y)$,

finite $U=\left\{s_{1}, s_{2}, \ldots, s_{n}\right\} \subseteq S$

$\exists x \in X: \inf \left\{g_{1}\left(s_{k} x\right) \mid g_{j} \in D, s_{k} \in U\right\}>1-\epsilon ;$

c. $\forall \in>0$, finite $D=\left\{g_{1}, g_{2}, \cdots, g_{m}\right\} \subseteq F(Y)$,

finite $U=\left\{s_{1}, s_{2}, \ldots, s_{n}\right\} \subseteq S$

$\exists x \in X: \frac{1}{n} \sum_{k=1}^{n} g_{i}\left(s_{k} x\right)>1-\epsilon(i=1, \ldots, m)$ and $\frac{1}{m} \sum_{i=1}^{m} g_{1}\left(s_{k} x\right)>1-\epsilon(k=1, \ldots, n)$;

d. $\exists \lambda \in \mathrm{MM}(\mathrm{F}), \forall s \in S, g \in F(y): \lambda\left(\mathrm{T}_{\mathrm{s}} \mathrm{g}\right)=1$ and $\lambda(\mathrm{g})=1$;

e. $\exists \mu \in M(F), \forall s \in S, g \in F(Y): \mu\left(T_{s} g\right)=1$ and $\mu(g)=1$;

f. $\exists \mu \in M(F), \forall v \in M(G), g \in F(Y): v \mu(g)=1$;

g. $\mathrm{Cle}(\mathrm{Y})$ contains a compact $\mathrm{MM}(\mathrm{G})$-invariant set;

h. $\forall \epsilon>0, g \in Z(Y)$, finite $U=\left\{s_{1}, s_{2}, \ldots, s_{n}\right\} \subseteq S \exists x \in X:\left|g\left(s_{k} x\right)\right|<\epsilon(k=1, \ldots, n)$;

i. $\quad \forall \epsilon>0$, finite $D=\left\{g_{1}, g_{2}, \ldots, g_{m}\right\} \subseteq Z(Y)$, finite $U=\left\{s_{1}, s_{2}, \ldots, s_{n}\right\} \subseteq S$;

$\exists x \in X: \sup \left\{\left|g_{j}\left(s_{k} x\right)\right| \mid g_{j} \in D, s_{k} \in U\right\}<\epsilon$ :

j. $\quad \forall \epsilon>0$, finite $D=\left\{g_{1}, g_{2}, \ldots, g_{m}\right\} \subseteq Z(Y)$. finite $U=\left\{s_{1}, s_{2}, \ldots, s_{n}\right\} \subseteq S$;

$\exists x \in X: \frac{1}{n} \sum_{x=1}^{n}\left|g_{1}\left(s_{k} x\right)\right|<\epsilon(i=1, \ldots . m)$ and $\frac{1}{m} \sum_{i=1}^{m}\left|g_{1}\left(s_{k} x\right)\right|<\epsilon(k=1, \ldots, n) ;$

k. $\exists \lambda \in M M(F), \forall s \in S . g \in Z(Y): \lambda\left(T_{s} g\right)=0$ and $\lambda(g)=0$;

1. $\exists \mu \in M(F), \forall s \in S, g \in Z(Y): \mu\left(T_{s} g\right)=0$ and $\mu(g)=0$;

m. $\exists \mu \in \mathrm{M}(\mathrm{F}), \forall v \in \mathrm{M}(\mathrm{G}), \mathrm{g} \in \mathrm{Z}(\mathrm{Y}): v \mu(\mathrm{g})=0$. 
PROOF: $\quad a-b: f(x)=\inf \left\{g_{1}(x) \mid g_{1} \in D\right\}$ is in $F(Y)$ because $0 \leq g_{i} \leq 1, g_{1} \equiv 1$ on $Y(i=1, \ldots, m)$. By (a) $\exists x \in X: f\left(s_{k} x\right)>1-\epsilon(k=1, \ldots, n)$. Because $U$ is finite, inf $\left\{f\left(s_{k} x\right) \mid s_{k} \in U\right\}>1-\epsilon$.

$$
b=c: \inf \left\{g_{1}\left(s_{k} x\right) \mid g_{1} \in D, s_{k} \epsilon U\right\}>1-\epsilon \rightarrow \sum_{k=1}^{n} g_{1}\left(s_{k} x\right) \geq n\left[\inf \left\{g_{1}\left(s_{k} x\right)\right\}\right]>n(1-\epsilon)
$$

and $\sum_{i=1}^{m} g_{1}\left(s_{k} x\right) \geq m\left[\inf \left\{g_{1}\left(s_{k} x\right)\right\}\right]>m(1-\epsilon)$.

$c \rightarrow d$ : For each $(\epsilon, U, D)$ in $(c)$ choose $x=x(\epsilon, U, D)$ so that $\frac{1}{n} \sum_{k=1}^{n} g\left(s_{k} x\right)$

$>1-\frac{1}{n} \epsilon(\forall g \in D)$. Let $r \in U, g \in D$. Then $g\left(s_{k} x\right) \leq 1(k=1, \ldots, n) \Rightarrow \sum_{s_{k} \neq r} g\left(s_{k} x\right) \leq n-1=-\sum_{s_{k} \neq r} g\left(s_{k} x\right)$

$z-n+1-g(r x)=\sum_{k=1}^{n} g\left(s_{k} x\right)-\sum_{s_{k} * r} g\left(s_{k} x\right)>1-\epsilon$. Define $(\epsilon, U, D) \leq\left(\epsilon^{\prime}, U^{\prime}, D^{\prime}\right)-$

$\epsilon z \epsilon^{\prime}, U \subseteq U^{\prime}, D \subseteq D^{\prime}$. The net $\langle e(x(\epsilon, U, D))\rangle \subseteq M M(F)$ has a subnet $\left\langle e\left(x_{m}\right)\right\rangle$ which $w^{*}$. converges to some $\lambda^{\prime} \epsilon \operatorname{MM}(F)$, since $M M(F)$ is compact. For $\delta>0$ and $(\epsilon, U, D) \geq(\delta,\{s\},\{g\})$ it follows that $1-\delta \leq 1-\epsilon<\mathrm{g}(\mathrm{sx}(\epsilon, \mathrm{U}, \mathrm{D}))=\mathrm{e}(\mathrm{x}(\epsilon, \mathrm{U}, \mathrm{D})) \mathrm{T}_{\mathrm{s}} \mathrm{g}$ by the earlier inequality. Therefore, $1-\delta \leq \lim _{m}\left[e\left(x_{m}\right)\left(T_{s} g\right)\right]=\left[\lim _{m} e\left(x_{m}\right)\right]\left(T_{s} g\right)=\lambda^{\prime}\left(T_{s} g\right)$. Since $\delta$ was arbitrary, $1 \leq \lambda^{\prime} T_{s} g$.

Because $0 \leq \mathrm{g} \leq 1, \mathrm{~T}_{\mathrm{s}} \mathrm{g} \leq 1$, and so $\lambda^{\prime}\left(\mathrm{T}_{\mathrm{s}} \mathrm{g}\right) \leq 1$. Thus, the first part of (d) is proven. Let $v \in M M(G)$; then $\lambda=v \lambda^{\prime} \in M M(F)$ and $\left(T_{\lambda^{\prime}} T_{s} g\right)(t)=\lambda^{\prime}\left[T_{t} T_{s} g\right]=\lambda^{\prime}\left(T_{s t} g\right)=1-\lambda\left(T_{s} g\right)=v \lambda^{\prime}\left(T_{s} g\right)=$ $v\left[T_{\lambda^{\prime}} T_{s} g\right]=v 1=1$; also $v \lambda^{\prime}(g)=v\left[T_{\lambda^{\prime}} g\right]=v 1=1$.

$d \rightarrow$ e: $M M(F) \subseteq M(F)$.

e $-\mathrm{f}$ : Let $v \in M(G)$ and $\mu$ be as in $(e)$, so that $\left(T_{\mu} g\right)(s)=\left(\mu T_{s} g\right)=1$; then $v \mu(\mathrm{g})=v\left(\mathrm{~T}_{\mu} \mathrm{g}\right)=v(1)=1$.

$f-$ a: We prove $($ not $(a)) \rightarrow($ not $(f))$. Suppose $\exists \epsilon>0, h \in F(Y), U=$ $\left\{s_{1}, s_{2}, \ldots, s_{n}\right\} \subseteq S$ such that $\forall x \in X, \exists s_{x} \in U: h\left(s_{x} x\right) \leq 1-\epsilon$. Define $v=\frac{1}{n} \sum_{k=1}^{n} e\left(s_{k}\right)$. Then $(\forall x \in X)$ [ve(x)]h $=\frac{1}{n} \sum_{k=1}^{n} h\left(s_{k} x\right) \leq 1-\epsilon / n$ because $0 \leq h \leq 1$ and, for some $s_{k}=s_{x}, h\left(s_{k} x\right) \leq 1-\epsilon$. This inequality, valid for all evaluations $\mathbf{e}(\mathbf{x})$, also holds for all finite means, and so for all limits $\mu \in M(F)$ of finite means: $v \mu(h) \leq 1-\frac{\epsilon}{n}$. Therefore $(f)$ is impossible.

$\mathrm{d} \sim \mathrm{g}$ : Choose $\lambda \in \mathrm{MM}(\mathrm{F})$ as in (d). $\mathrm{MM}(\mathrm{G}) \lambda$ is then an $\mathrm{MM}(\mathrm{G})$-invariant set. Since $\mathrm{Cl}[\mathrm{e}(\mathrm{Y})]$ is closed, it suffices to show that $\mathrm{e}(\mathrm{s}) \lambda_{\epsilon} \mathrm{Cl}[\mathrm{e}(\mathrm{Y})]$ for $\forall \mathrm{seS}$. Suppose that $\exists \mathrm{s}_{0}$ : $\mathrm{e}\left(\mathrm{s}_{0}\right) \lambda \notin \mathrm{Cl}[\mathrm{e}(\mathrm{Y})]$. Then, since $\mathrm{MM}(\mathrm{F})$ is compact Hausdorff and so completely regular, $\exists h \in C(M M(F)): 0 \leq h \leq 1, h\left(e\left(s_{0}\right) \lambda\right)=0$, and $h(C l[e(Y)])=1 . g=h \propto \epsilon F(Y)$ because for $y \in Y$ $\mathrm{g}(\mathrm{y})=\mathrm{h}(\mathrm{e}(\mathrm{y}))=1$. Then $\lambda\left(\mathrm{T}_{\mathrm{s}_{0}} \mathrm{~g}\right)=\left[\mathrm{e}\left(\mathrm{s}_{0}\right) \lambda\right] \mathrm{g}=\mathrm{h}\left(\mathrm{e}\left(\mathrm{s}_{0}\right) \lambda\right)=0$, contradicting (d).

$\mathrm{g} \rightarrow \mathrm{d}$ : Let $\mathrm{I}$ be an $\mathrm{MM}(\mathrm{G})$-invariant set $\varsigma \mathrm{Cl}(\mathrm{e}(\mathrm{Y}))$. If $\lambda \in \mathrm{I}$, then $\mathrm{e}(\mathrm{s}) \lambda \in \mathrm{I} \_\mathrm{Cl}(\mathrm{e}(\mathrm{Y}))(\forall s \in \mathrm{S})$. Therefore, $\lambda\left(\mathrm{T}_{\mathrm{s}} \mathrm{g}\right)=[\mathrm{e}(\mathrm{s}) \lambda] \mathrm{g}=1(\forall \mathrm{geF}(\mathrm{Y}))$. Clearly $\lambda(\mathrm{g})=1$ $(\forall g \in F(Y))$. 
$a \rightarrow h$ : Assume $Y$ is $F, S$-thick in $X$. Let $\epsilon>0, g \in Z(Y)$, finite UcS. If $g=0$, result is trivial; hence, assume that $g \bowtie 0$. Then $1-\frac{1}{\|g\|}|g| \epsilon F(Y)$. Consequently, $\exists x \in X$ : $1-\frac{1}{\|g\|}\left|g\left(s_{k} x\right)\right| \geq 1-\frac{\epsilon}{\|g\|}$, whence $\left|g\left(s_{k} \mathbf{x}\right)\right|<\epsilon(k=1, \ldots . n)$.

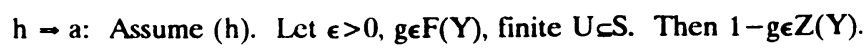

Therefore. $\exists x \epsilon X:\left|1-g\left(s_{k} x\right)\right|<\epsilon--\epsilon<1-g\left(s_{k} x\right)<\epsilon--g\left(s_{k} x\right)<-1+\epsilon-g\left(s_{k} x\right)>1-\epsilon(k=1 \ldots, n)$.

$h-i: \sup \left\{\left|g_{j}\right| \mid g_{j} \in D\right\} \in Z(Y)$, because $g_{j} \equiv 0$ on $Y(j=1 \ldots . . m)$.

$i-k$ : For each $(\epsilon, U, D)$ in (i) choose $x=x(\epsilon, U, D)$. Define $(\epsilon, U, D) \leq\left(\epsilon^{\prime}, U^{\prime}, D^{\prime}\right)-\epsilon z \epsilon^{\prime}, U \subseteq U^{\prime}, D \in D^{\prime}$. The net $\langle e(x(\epsilon, U, D))>\subseteq M M(F)$ has a subnet $\left\langle e\left(x_{m}\right)\right\rangle$ which converges to some $\lambda \epsilon M M(F)$ since $M M(F)$ is compact. Let $\delta>0$. If $(\epsilon, U, D) \geq$ $(\delta,\{s\},\{g\})$, then $\delta z \epsilon>\sup \left\{\left|g_{j}\left(s_{k} x(\epsilon, U, D)\right)\right| \mid g_{j} \epsilon D, s_{k} \epsilon U\right\} z|g(s x(\epsilon, U, D))|$. Ergo $\delta z$ $\lim _{m}\left[c\left(x_{m}\right)\left|T_{s} g\right|\right]=\left[\lim _{m} c\left(x_{m}\right)\right]\left|T_{s} g\right|=\lambda\left|T_{s} g\right|$. Since $\delta$ was arbitrary, the first part of $(k)$ is proven. The second part is shown in the same manner as the second part of $(c)-(d)$.

$\mathrm{i} \rightarrow \mathrm{j}$ : Trivial.

$j-i$ : In the first part of $(j)$, replace $\epsilon$ by $\frac{\epsilon}{n}: \frac{\epsilon}{n}>\frac{1}{n} \sum_{k=1}^{n}\left|g_{j}\left(s_{k} x\right)\right|(j=1, \ldots, n)-$ $\epsilon>\sum_{k=1}^{n}\left|g_{j}\left(s_{k} x\right)\right|>\sup \left\{\left|g_{j}\left(s_{k} x\right)\right| \mid g_{j} \in D, s_{k} \in U\right\}$

$\mathrm{k} \rightarrow \mathrm{l}, \mathrm{l} \rightarrow \mathrm{m}:$ Trivial.

$m-h$ : We show $(\operatorname{not}(h)) \Rightarrow(\operatorname{not}(m))$. Suppose $\exists \epsilon>0, h \in Z(Y)$, finite UєS

such that $\forall x \in X, \exists s_{x} \in U:\left|h\left(s_{x} x\right)\right| z \epsilon$. Define $v=\frac{1}{n} \sum_{x=1}^{n} e\left(s_{k}\right)$. Then $\forall x \in X:\{v(e(x))]|h|=$ $\frac{1}{n} \sum_{k=1}^{n}\left|h\left(s_{k} x\right)\right| z \epsilon / n$, because $|h| \geq 0$ and for some $s_{k}=s_{x},\left|h\left(s_{k} x\right)\right| \geq \epsilon$. Hence, replacing $e(x)$ by any finite mean, then for any $\mu \in \mathrm{M}(\mathrm{F}), \nu \mu|\mathrm{h}| z \epsilon / \mathrm{n}$. Therefore (m) is impossible.

Remark 3.4. Parts d., e., k., and l., of Theorem 3.3 suggest that $S$ behaves with regard to thickness as though it contained an identity. In fact, if $S^{1}$ denotes the semigroup $S$ with a discrete identity 1 adjoined, then $Y$ is $F, S$-thick in $X-Y$ is $F, S^{1}$-thick in $X$ where $S^{1}$ acts on $X$ in the natural way.

Corollary 3.5. If the characteristic function $\chi_{Y} \in F$, then the following statements are equivalent:
a. $\mathrm{Y}$ is F,S-thick in $\mathrm{X}$;
b. $\quad \forall$ finite $U=\left\{s_{1}, s_{2}, \ldots, s_{n}\right\} \subseteq S, \exists x \in X: s_{k} x \in Y(k=1, \ldots, n)$;
c. $\quad \forall$ finite $U=\left\{s_{1}, s_{2}, \ldots, s_{n}\right\} \subseteq S, \exists y \in Y: s_{k} y \in Y(k=1, \ldots, n)$;
d. The family $\left\{\mathrm{s}^{-1} \mathrm{Y} \mid \mathrm{se} S\right\}$ has the finite intersection property;
e. $\bigcap_{s \in S} \mathrm{Cl} e\left(s^{-1} Y\right) \neq \emptyset$ where $e\left(s^{-1} Y\right)=\{e(x) \mid s x \in Y\}$.

PROOF: $\quad \mathrm{e}-\mathrm{a}$ : Let $\mu \epsilon \bigcap_{s \in S} \mathrm{Cl} e\left(\mathrm{~s}^{-1}\right) \mathrm{Y}$; also let $s \in \mathrm{S}$. $g \in \mathrm{F}(\mathrm{Y})$. Then $\mu \in \mathrm{Cl} \mathrm{e}\left(\mathrm{s}^{-1} \mathrm{Y}\right)$, so $\exists$ net $\left\langle x_{n}\right\rangle$ such that $\mu=w^{*}-\lim e\left(x_{n}\right)$ and $s_{n} \in Y(\forall n)$; whence $\mu T_{s} g=\left[w^{*}-\lim _{n} e\left(x_{n}\right)\right] T_{s} g=$ $\lim _{n}\left[g\left(s x_{n}\right)\right]=\lim 1_{n}=1$. Now let $\lambda \in M(G)$. Then $\lambda \mu \in M(F)$ and $\lambda \mu T_{s} g=\lambda\left[T_{\mu}\left(T_{s} g\right)\right]=$ 
$\lambda\left[L_{s} T_{\mu} g\right]=\lambda\left[L_{s} l\right]=1 ;$ also $\lambda \mu(g)=\lambda\left[T_{\mu} g\right]=\lambda\left[\mu T_{(\bullet) g]} g\right][1]=1$. Therefore by 3.3.e $Y$ is F,S-thick.

Results for transformation semigroups comparable to the theorems of section 1 can be generalized in the same way as in [Junghenn 1979, p. 40, theorem 2].

Theorem 3.6. Let $\langle\mathrm{S}, \mathrm{X}\rangle$ be a transformation semigroup;

$\langle T, Y\rangle$ be a subtransformation semigroup of $\langle\mathrm{S}, \mathrm{X}\rangle$; and

$\mathrm{F} \subseteq \mathrm{B}(\mathrm{X})$ be a translation invariant, conjugate-closed, norm-closed subalgebra which contains the constant functions.

If $F$ has invariant mean $\mu$ with respect to $\langle T, X\rangle$ such that inf $\{\mu(g) \mid g \in F(Y)\}>0$, then $\left.F\right|_{Y}$ has invariant mean with respect to $\langle T, Y\rangle$.

PROOF: $X$ is embedded in the compact set $M M(F)$ by $e(\bullet)$, and $F-C(M M(F))$ by the Gelfand representation theorem. Also $\mathrm{Cl}(\mathrm{Y}) \subseteq \mathrm{MM}(\mathrm{F})$. By the Riesz representation theorem, the invariant mean $\mu$ defines a regular Borel probability measure $\hat{\mu}$ on $\operatorname{MM}(F)$ such that $\mu(f)=$ $\int_{M M(F)} \hat{d} \hat{\mu}(\forall \hat{f} \epsilon F)$. Invariance of $\mu$ is reflected in $\hat{\mu}$ as follows:

$$
\int_{M M(F)} T_{e(t)} \hat{f} d \hat{\mu}=\int_{M M(F)} \hat{T} \hat{f} d \hat{\mu}=\mu\left(T_{t} f\right)=\mu(f)=\int_{M M(F)} \hat{f} d \hat{\mu}\left(H_{\epsilon} T\right)
$$

Since $\mu$ is regular, $\hat{\mu}(\mathrm{Cl} e(Y))=\inf \{\hat{\mu}(U) \mid U$ open, $\mathrm{Cl} e(Y) \leq U\}$. Now let $U$ be any open set such that $\mathrm{Cl} e(\mathrm{Y}) \subseteq U$. Because $\mathrm{MM}(\mathrm{F})$ is normal, by Urysohn's lemma, $\exists \hat{\mathrm{g}} \in \mathrm{C}(\mathrm{MM}(\mathrm{F}))-\mathrm{F}$ such that $\hat{\mathrm{g}}(\mathrm{Cl} e(\mathrm{Y})) \equiv 1, \widehat{\mathrm{g}}\left(\mathrm{U}^{\mathrm{c}}\right) \equiv 0$, and $0 \leq \hat{\mathrm{g}} \leq 1$; thus $\hat{\mathrm{g}} \leq \chi_{\mathrm{U}}$ and $\mathrm{g}$, the correlative of $\hat{\mathrm{g}}$, is in $F(Y) . \mu(g)=\int_{M M(F)} \hat{g} d \hat{\mu} \leq \int_{M M(F)} \chi_{U}^{d \hat{\mu}}=\hat{\mu}(U)$. Therefore by hypothesis $0<$ $\inf \{\boldsymbol{\mu}(\mathrm{g}) \mid \mathrm{g} \in \mathrm{F}(\mathrm{Y})\} \leq \inf \{\hat{\boldsymbol{\mu}}(\mathrm{U}) \mid \mathrm{U}$ open, $\mathrm{Cl} \mathrm{e}(\mathrm{Y}) \subset \mathrm{U}\}=\hat{\mu}(\mathrm{Cl} \mathrm{e}(\mathrm{Y}))$. Ergo, $v(f)=\frac{1}{\hat{\mu}(C l e(Y))} \int_{C l e(Y)} \hat{f} d \hat{\mu}$ is a mean on $F$.

Define $v_{0}$ on $\left.F\right|_{Y}$ by $v_{0}\left(\left.f\right|_{Y}\right)=v(f) . \quad v_{0}$ is wcll-defined because $\left.\left.f\right|_{Y} \equiv g\right|_{Y}-f-g \in Z(Y)$. $\widehat{(f-g)} \equiv 0$ on $\mathrm{Cl} \mathrm{e}(\mathrm{Y})-0=v(\mathrm{f}-\mathrm{g})=v(\mathrm{f})-v(\mathrm{~g})$. Also $v_{0} \in \mathrm{M}(\mathrm{F} / \mathrm{Y})$.

To show that $v_{0}$ is invariant it suffices to prove that $\int_{C l e(Y)} T_{e(t)} \hat{\mathrm{f} d} \hat{\mu}=\int_{\mathrm{Cle}(\mathrm{Y})} \hat{\mathrm{d}} \hat{\mu} \hat{\mathrm{H}}(\forall t \in T)$.

Fix teT. Define $E_{1}=e(t)^{-1}(C l e(Y)) \backslash C l e(Y), E_{n}=e(t)^{-1}\left(E_{n-1}\right)(n \geq 2)$. The $E_{n}$ are pairwise disjoint: $\mu \in E_{2}-e(t) \mu \in E_{1}-e(t) \mu \notin C l e(Y) \rightarrow \mu \notin E_{1}$, so $E_{1} \cap E_{2}=0$. Assume that $E_{m}$ and $E_{n}$ are pairwise disjoint $(1 \leq m<n)$. Then $\mu \in E_{n+1}-e(t) \mu \in E_{n}-e(t) \mu \in E_{m}(1 \leq m<n)$ $\rightarrow \mu \notin(t)^{-1} E_{m}=E_{m+1}=E_{p}(2 \leq p=m+1<n+1)$, so $E_{n+1} \cap E_{p}=0$. Also $\mu \in E_{n+1}-e(t)^{n} \mu \in E_{1}$ (by induction) $-\mathrm{e}(\mathrm{t})^{\mathrm{n}} \mu \notin \mathrm{Cl} \mathrm{e}(\mathrm{Y})$, but $\mu \epsilon \mathrm{E}_{1}-\mathrm{e}(\mathrm{t}) \mu \epsilon \mathrm{Cl} \mathrm{e}(\mathrm{Y})-\mathrm{e}(\mathrm{t})^{\mathrm{n}} \mu \epsilon \mathrm{CL} \mathrm{e}(\mathrm{Y})$ (by invariance of $Y)$, so $E_{n+1} \cap E_{1}=\emptyset$. The $E_{n}$ are Borel sets since $\mu \rightarrow e(t) \mu$ is $w^{*}=$ continuous for $\forall \mu \in M M(F)$.

Because $(\forall n \geq 2) T_{e(t)} \chi_{E_{n-1}}(\mu)=\chi_{E_{n-1}}(e(t) \mu)=\chi_{e(t)} E_{n-1}(\mu)$, it follows that $\hat{\mu}\left(E_{n}\right)=\hat{\mu}\left(e(t)^{-1} E_{n-1}\right)=\int_{M M(F)} x_{e(t)^{-1} E_{n-1}} d \hat{\mu}=\int_{M M(F)} T_{e(t)} x_{E_{n-1}} d \hat{\mu}=\int_{M M(F)} x_{E_{n-1}} d \hat{\mu}=\hat{\mu}\left(E_{n-1}\right)$. Therefore, $1 \geq \hat{\mu}\left(E_{1} \cup E_{2} \cup \ldots \cup E_{n}\right)=\sum_{j=1}^{n} \hat{\mu}\left(E_{j}\right)=n \hat{\mu}\left(E_{1}\right)$. Since this holds for arbitrary $n$, $\hat{\boldsymbol{\mu}}\left(\mathrm{E}_{1}\right)=\mathbf{0}$.

Because $Y$ is invariant, $e(T) C l e(Y) \_C l e(Y)$, whence $C l e(Y) \backslash e(t)^{-1} C l e(Y)=0$. Since

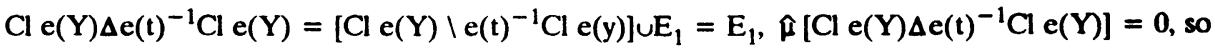
$\int_{C l e(Y)} T_{e(t)} \hat{f} d \hat{\mu}=\int_{e(t)^{-1} C l e(Y)} T_{e(t)} \hat{f} d \hat{\mu}=\int_{M M(F)} T_{e(t)}\left[\hat{f} x_{C l e(Y)}\right] d \hat{\mu}=\int_{C l e(Y)} \hat{f} d \hat{\mu}$. 
Theorcm 3.7. Let $\langle S, X\rangle$ be a $\tau$-semigroup;

$\langle T, Y\rangle$ be a sub $\tau$-semigroup of $\langle S, X\rangle$;

$\mathrm{F} \subset \mathrm{B}(\mathrm{X})$ be a translation invariant, norm-closed, $\mathrm{G}$-introverted subalgebra which contains the constant functions.

1. If $\left.F\right|_{Y}$ has an invariant mean with respect to $\langle T, Y\rangle$ and $T$ is $G$-thick in $S$. then $F$ has an invariant mean with respect to $\langle\mathrm{S}, \mathrm{X}\rangle$.

2. If $G$ has a left-invariant mean and $Y$ is $F$.S-thick in $X$, then $F / Y$ has an invariant mean with respect to $\langle T, Y\rangle$.

PROOF: 1. Functional $\bar{\mu}$ in $\left.F\right|_{Y^{*}}$ defines a functional $\mu$ in $F^{*}$ by $\mu f=\left.\bar{\mu} f\right|_{Y}(\forall f \in F)$, thus $\mu T_{t} f=\left.\bar{\mu} T_{t} f\right|_{Y}(\forall f \epsilon F, t \epsilon T)$. Therefore, because $F$ is $G$-introverted. $\left.F\right|_{Y}$ is $\left.G\right|_{T}$-introverted.

Relative to the algebra $\left.F\right|_{Y}$ defined on $\langle T, Y\rangle$ : Let $\bar{\mu}$ be an invariant mean of $\left.F\right|_{Y}$; then $\mathrm{e}(\mathrm{t}) \bar{\mu}=\bar{\mu}\left(\mathrm{T}_{\mathrm{s}} \bullet\right)=\bar{\mu}(\forall t \epsilon T)$ where $\mathrm{e}(\mathrm{t}) \epsilon \mathrm{MM}\left(\left.\mathrm{G}\right|_{\mathrm{T}}\right)$. Let $\bar{\lambda} \epsilon \mathrm{Cl} \mathrm{e}(\mathrm{T})=\mathrm{MM}\left(\left.\mathrm{G}\right|_{\mathrm{T}}\right)$, and let $\left\langle e\left(t_{\alpha}\right)\right\rangle=e(T) \subseteq M M\left(\left.G\right|_{T}\right)$ be a net such that $\bar{\lambda}=w^{*}-\lim e\left(t_{\alpha}\right)$. Ergo,

$\left.\bar{\lambda} \bar{\mu}=\mid w *-\lim _{\alpha} e\left(t_{\alpha}\right)\right] \bar{\mu}=\lim _{\alpha}\left[e\left(t_{\alpha}\right) \bar{\mu}\right]=\lim _{\alpha} \bar{\mu}=\bar{\mu}$. That is, $\bar{\lambda} \bar{\mu}=\bar{\mu}(\forall \bar{\lambda} \epsilon \mathrm{Cl} e(T))$.

Relative to the algebra $F$ defined on $\langle S, X\rangle: \exists$ left-ideal $\mathrm{K}$ of $\mathrm{Cl}$ e(S) in $\mathrm{Cl} e(\mathrm{~T}) \subset \mathrm{MM}(\mathrm{G})$ [Wilde \& Witz, 1967, lemma 5.1]. Choose $\lambda_{0} \in \mathrm{K}$. Then e(s) $\lambda_{0} \in \mathrm{K} \_\mathrm{Cl}$ e(T) $\subset \mathrm{MM}(\mathrm{G})(\forall \mathrm{s} \in \mathrm{S})$.

Any $\lambda \epsilon \mathrm{Cl} e(T) \subset M M(G)$ gives rise to a $\bar{\lambda} \epsilon \mathrm{Cl} e(T) \subset M M\left(\left.G\right|_{T}\right)$ in the following way: $\lambda^{\prime}=w^{*}-\lim _{\alpha} e\left(t_{\alpha}\right) \epsilon M M(G)$. Now $\left\langle e\left(t_{\alpha}\right)\right\rangle$ is a net in $e(T) \subset M M\left(\left.G\right|_{T}\right)$ so has a convergent subnet $\left\langle e\left(t_{\beta}\right)\right\rangle$ with $\bar{\lambda}=w^{*}-\lim e\left(t_{\beta}\right) \epsilon M M\left(\left.G\right|_{T}\right)$. $\bar{\lambda}$ may not be unique. For $\bar{\mu} \epsilon \mathrm{F} \mid \mathrm{Y}^{*}$ define $\mu \epsilon \mathrm{F}^{*}$ by $\mu f=\left.\bar{\mu} f\right|_{Y}(\forall f \in F)$ as we have done earlier. Then for all $\left.f \in F \bar{\lambda} \bar{\mu} f\right|_{Y}=\bar{\lambda}\left(\left.T_{\bar{\mu}} f\right|_{Y}\right)=$ $\lim _{\beta}\left[\left.c\left(t_{\beta}\right) T_{\bar{\mu}} f\right|_{Y}\right]=\lim _{\beta}\left[\left.\bar{\mu} T_{t_{B}} f\right|_{Y}\right] ;$ also. $\lambda_{\mu} f=\lambda\left(T_{\mu} f\right)=\lim _{\alpha}\left[\left.\bar{\mu} T_{t_{\alpha}} f\right|_{Y}\right] ; \operatorname{ergo} \lambda \mu(f)=$ $\bar{\lambda} \bar{\mu}\left(\left.f\right|_{Y}\right)$, regardless of the choice of $\bar{\lambda}$ which is associated with $\lambda$.

Finally, choose $\bar{\mu}$ to be an invariant mean of $\left.F\right|_{Y}$, and define $\mu \in M(F)$ as before. Then $\lambda_{\mu}(f)$ $=\bar{\lambda} \bar{\mu}\left(\left.f\right|_{Y}\right)=\bar{\mu}\left(\left.f\right|_{Y}\right)=\mu(f)$, that is, $\lambda_{\mu}=\mu\left(\forall \lambda_{\epsilon} C l e(T)=M M(G)\right)$. In particular, $c(s) \lambda_{0} \mu=$ $\mu(\forall s \in S)$, so that $\lambda_{0} \mu$ is invariant.

2. Because $Y$ is $F, S-t h i c k$ in $X$, then by Theorem 3.3.f $\exists \mu \in M(F)$ such that $v \mu(f)=1(\forall v \in M(G), f \in F(Y))$. Let $v$ be an invariant mean of $G$. Then $v \mu$ is an invariant mean of $F$ such that $v \mu(f)=1(\forall f \in F(Y))$. By Theorem 3.6 F/ has an invariant mean with respect to $\langle\mathrm{T}, \mathrm{Y}\rangle$.

QED

In the preceding theorem the thickness condition on $\mathrm{T}$ in (1) implies the thickness condition on $\mathrm{Y}$ in (2) according to the following lemma:

Lemma 3.8. Let $\langle S, X\rangle$ be a $\tau$-semigroup;

$\langle\mathrm{T}, \mathrm{Y}\rangle$ be a sub $\tau$-semigroup of $\langle\mathrm{S}, \mathrm{X}\rangle$;

$F \subseteq B(X)$ be a translation-invariant, norm-closed, $G$-introverted subalgebra which contains the constant functions.

If $T$ is G-thick in $S$, then $Y$ is F.S-thick in $X$.

PROOF: Let $f \in F(Y)$ : $0 \leq f \leq 1, f \equiv 1$ on $Y$. Then $T_{e(y)} f \in F(T)(\forall y \in Y)$. By Theorem 3.3.e applied to $L(S, G) \exists \mu \in M(G)$ such that $1=\mu\left(L_{s} T_{e(y)} f\right)=\mu\left(T_{e(y)} T_{s} f\right)=\mu e(y) T_{s} f$ and $1=$ $\mu T_{e(y)} f=\mu e(y) f$. Then $\mu \mathrm{e}(y) \epsilon M(F)$ has the properties required by Theorem 3.3.e for $Y$ to be F,S-thick.

QED

Junghenn's theorem of section 1 is obtained from Theorem 3.7 and Lemma 3.8 by letting $\mathrm{X}=\mathrm{S}, \mathrm{Y}=\mathrm{T}$, and the action be left multiplication.

\section{Multiplicative Means and Thickness}

Several results connect multiplicative means with thickness. $F$ is assumed to be an $S$. invariant, norm-closed algebra $\_\mathrm{C}(\mathrm{X})$ which contains the constant functions. 
Thoorcm 4.1 If $F$ has an invariant multiplicative mean, then for any finite partition $\left\{A_{i}\right\}_{1}^{n}$ of $X \exists k$ such that $A_{k}$ is F.S-thick.

PROOF: Let $v \in M M(F)$ be invariant. $v$ induces a regular Borel probability measure $\diamond$ defined on $\operatorname{MM}(F)$, and $\sum_{1}^{n} \hat{v}\left(\operatorname{Cl} e\left(A_{1}\right)\right) \geq 1$. Because $v$ is multiplicative, for each $i \hat{v}\left(\mathrm{Cl} e\left(A_{i}\right)\right)$ $=0$ or $\hat{v}\left(\mathrm{Cl} e\left(A_{1}\right)\right)=1$. Hence. $\exists k$ such that $\widehat{v}\left(\mathrm{Cl} e\left(A_{k}\right)\right)=1$. Therefore, $v(f)=1\left(\forall f \in F\left(A_{k}\right)\right)$ becausc $\chi_{A_{k}} \leq f \leq 1-X_{C l e\left(A_{k}\right)} \leq \hat{f} \leq 1$ and $1=\hat{v}\left(C l e\left(A_{k}\right)\right)=\int \chi_{C l e\left(A_{k}\right)} d \hat{v} \leq \int \hat{f} d \hat{v}=v(f) \leq 1$.

Then, by Theorem 3.3.d $A_{k}$ is F.S-thick.

Definition 4.2 $K(f, s)=\left\{\mu \in M M(F) \mid \mu\left(T_{s} f-f\right)=0\right\}$

Theorem 4.3. The following are equivalent:

a. F has an invariant multiplicative mean;

b. It is not the case that $M M(F) \subseteq \bigcup_{\substack{f \in F \\ s \in S}} K^{c}(f, s)$ :

c. It is not the case that $\exists f_{1}, \ldots, f_{n} \in F ; \exists s_{1}, \ldots, s_{n} \in S: M M(F) \subseteq \bigcup_{i=1}^{n} K^{c}\left(f_{1}, s_{1}\right)$;

d. $\forall f_{1}, \ldots, f_{n} \in F ; \forall s_{1}, \ldots, s_{n} \in S ; \forall 8>0 ; \exists x_{\delta}: e\left(x_{\delta}\right) \sum_{i=1}^{n}\left|T_{s_{1}} f_{1}-f_{1}\right|<8$;

e. $\forall \mathrm{f}_{1}, \ldots, \mathrm{f}_{\mathrm{n}} \in \mathrm{F} ; \forall \mathrm{s}_{1}, \ldots, \mathrm{s}_{\mathrm{n}} \in \mathrm{S} ; \forall \delta>0, \exists \mathrm{x}_{\delta}: \mathrm{T}_{\mathrm{S}_{1}} \mathrm{f}_{1}\left(\mathrm{x}_{\delta}\right)-\mathrm{f}_{1}\left(\mathrm{x}_{\delta}\right) \mid<\delta(\mathrm{i}=1, \ldots, \mathrm{n})$;

f. $\forall f_{1}, \ldots, f_{n} \in F ; \forall s_{1}, \ldots, s_{n} \in S: \exists \lambda \in M M(F): \lambda\left|T_{\varsigma_{1}} l_{1}-f_{1}\right|=0 \quad(i=1, \ldots, n)$;

g. $\forall \mathrm{f}_{1}, \ldots, \mathrm{f}_{\mathrm{n}} \in \mathrm{F} ; \forall \mathrm{s}_{1}, \ldots, \mathrm{s}_{\mathrm{n}} \in \mathrm{S} ; \exists \lambda \in \mathrm{MM}(\mathrm{F}): \lambda\left(\mathrm{T}_{\mathrm{s}_{1}} \mathrm{f}_{1}-\mathrm{f}_{1}\right)=0 \quad(\mathrm{i}=1, \ldots, \mathrm{n})$;

h. $\forall \epsilon>0 ; \forall f_{1}, \ldots, f_{n} \in F ; \forall s_{1}, \ldots, s_{n} \in S: \exists c_{1}, \ldots, c_{n} \in C ; \exists Y \subset X:\left|f_{k}-c_{k}\right|<\epsilon$ and $\left|T_{s_{k}} f_{k}-c_{k}\right|<\epsilon$ on $Y(k=1, \ldots, n)$ and $Y$ is F,S-thick in $X$.

PROOF: $a-b$ : $F$ has an invariant multiplicative mean $-\exists \lambda \in M M(F): \lambda \in K(f, s)(\forall f \in F, s \in S)-$ the $K^{c}(f, s)$ do not cover all of $\mathrm{MM}(\mathrm{F})$.

$\sim \mathrm{b} \sim \sim \mathrm{c}: \mathrm{MM}(\mathrm{F})$ is compact and the $\mathrm{K}^{\mathrm{c}}(\mathrm{f}, \mathrm{s})$ are open.

$\sim c \rightarrow \sim d$ : Let $f_{1}, \ldots, f_{n} \in F$ and $s_{1}, \ldots, s_{n} \in S$ be as in the negation of (c). If for any $8>0 \exists x_{\delta} \in X$ such that $e\left(x_{\delta}\right) \sum\left|T_{s_{k}} f_{k}-f_{k}\right|=\sum\left|T_{s_{k}} f_{k}\left(x_{\delta}\right)-f\left(x_{\delta}\right)\right|<\delta$, then the net $\left\langle e\left(x_{\delta}\right)\right\rangle_{\delta>0} \subseteq M M(F)$ contains a convergent subnet $\left\langle e\left(x_{\delta_{\alpha}}\right)\right\rangle_{\alpha \epsilon A}$ of $\left\langle e\left(x_{\delta}\right)\right\rangle$ and $w *-\lim _{\alpha} e\left(x_{\delta}\right)=\lambda \epsilon M M(F) ;$ thus, for any $\epsilon>0 \exists \alpha_{0} \in A: \alpha \geq \alpha_{0}-\left|\lambda \sum\right| T_{s_{k}} f_{k}-f_{k} \mid-$ $e\left(x_{\delta_{\varepsilon}}\right) \sum\left|T_{s_{k}} f_{k}-f_{k}\right| \mid<\frac{\epsilon}{2}$. Let $\alpha_{1} \epsilon A$ be $2 \alpha_{0}$ and such that $\delta_{\alpha_{1}}<\frac{\epsilon}{2}$, so that $e\left(x_{\delta_{\varepsilon_{1}}}\right) \sum\left|\mathrm{T}_{\mathrm{s}_{\mathrm{k}}} \mathrm{f}_{\mathrm{k}}-\mathrm{f}_{\mathrm{k}}\right|<\frac{\epsilon}{2}$. Then $0 \leq \lambda \sum\left|\mathrm{T}_{\mathrm{s}_{\mathrm{k}}} \mathrm{f}_{\mathrm{k}}-\mathrm{f}_{\mathrm{k}}\right|<\mathrm{e}\left(\mathrm{x}_{\delta_{\boldsymbol{e}_{1}}}\right) \cdot \sum\left|\mathrm{T}_{\mathrm{s}_{\mathrm{k}}} \mathrm{f}_{\mathrm{k}}-\mathrm{f}_{\mathrm{k}}\right|+$ $\frac{\epsilon}{2}<\frac{\epsilon}{2}+\frac{\epsilon}{2}=\epsilon$. Since $\epsilon$ was arbitrary. $\lambda \sum\left|T_{s_{l}} f_{k}-f_{k}\right|=0 \Rightarrow\left|T_{s_{k}} f_{k}-f_{k}\right|=$ $0(\forall k)-\lambda\left(T_{s_{k}} f_{k}-f_{k}\right)=0(\forall k)$. The last equation contradicts that $\lambda \epsilon \bigcup_{1=1}^{n} K^{c}\left(f_{i}, s_{1}\right)$. 
$\sim d-\sim c$ : Suppose that $\exists f_{1}, \ldots, f_{n} \in F$ and $s_{1}, \ldots, s_{n} \in S$ and $\delta>0$ such that

$(\forall x) e(x) \sum\left|T_{s_{k}} f_{k}-f_{k}\right| \geq \delta$. Let $\lambda \in M M(F)$, so that $\lambda=w^{*}-\lim e\left(x_{v}\right)$ with $x_{v} \in X(\forall v)$.

Then $\lambda \sum\left|\mathrm{T}_{\mathrm{s}_{\mathrm{k}}} \mathrm{f}_{\mathrm{k}}-\mathrm{f}_{\mathrm{k}}\right|=w *-\lim \mathrm{c}\left(\mathrm{x}_{\mathrm{v}}\right) \sum\left|\mathrm{T}_{\mathrm{s}_{\mathrm{k}}} \mathrm{f}_{\mathrm{k}}-\mathrm{f}_{\mathrm{k}}\right| \geq 8-\exists \mathrm{k}^{0}$ such that

$\frac{\delta}{\mathrm{n}} \leq \lambda\left|\mathrm{T}_{\mathrm{s}_{\mathrm{k}^{0}}} \mathrm{f}_{\mathrm{k}^{0}}-\mathrm{f}_{\mathrm{k}^{0}}\right|=\left|\lambda\left(\mathrm{T}_{\mathrm{s}_{\mathrm{k}^{0}}} \mathrm{f}_{\mathrm{k}^{0}}-\mathrm{f}_{\mathrm{k}^{0}}\right)\right|(\lambda|\mathrm{g}|=|\lambda \mathrm{g}|$ because $\lambda$ is multiplicative $)$

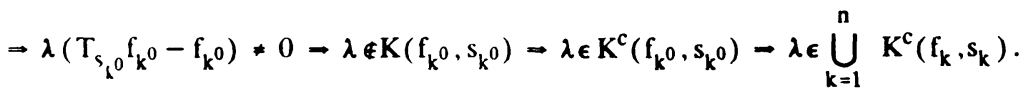

$\mathrm{e} \Rightarrow \mathrm{f}:\left\langle e\left(x_{\delta}\right)\right\rangle_{\delta>0}$ is a net in $\mathrm{MM}(\mathrm{F})$ so has a convergent subnet $\left\langle e\left(x_{\delta_{\alpha}}\right)\right\rangle_{\alpha \in A}$. Let $\lambda$ denote the $w^{*}-$ limit of $\left\langle e\left(x_{\delta_{\alpha}}\right)\right\rangle$. Then by the same reasoning as in $\sim c-\sim d, \exists \alpha_{1} \in A$ such that $0 \leq \lambda\left|\mathrm{T}_{s_{k}} \mathrm{f}_{k}-\mathrm{f}_{\mathrm{k}}\right|<\mathrm{e}\left(\mathrm{x}_{\delta_{\mathrm{e}_{1}}}\right)\left|\mathrm{T}_{\mathrm{s}_{\mathrm{k}}} \mathrm{f}_{\mathrm{k}}-\mathrm{f}_{\mathrm{k}}\right|+\frac{\epsilon}{2}<\frac{\epsilon}{2}+\frac{\epsilon}{2}=\epsilon$. Since $\epsilon$ is arbitrary. $\lambda\left|T_{s_{k}} f_{k}-f_{k}\right|=0$.

$f \rightarrow e$ : Since $\lambda \in \operatorname{MM}(F), \lambda=w^{*}-\lim e\left(x_{v}\right)$ for some net $\left\langle e\left(x_{v}\right)\right\rangle$ with $x_{v} \in X(\forall v)$. By the definition of $w^{*}$-convergence, for any $\delta>0 \exists e\left(x_{\delta}\right) \epsilon\left\langle e\left(x_{v}\right)\right\rangle$ such that $e\left(x_{\delta}\right)\left|T_{s_{1}} f_{1}-f_{i}\right|<\delta(i=1, \ldots, n)$.

$a=h$ : Assume (a) and let $f_{1}, \ldots, f_{n} \in F ; s_{1}, \ldots, s_{n} \in S ;$ and $\epsilon>0$.

Notation: $L\left(r_{1}, \ldots, r_{n}\right)=f^{-1}\left(S_{\epsilon}\left(r_{1}\right)\right) \cap f_{2}^{-1}\left(S_{\epsilon}\left(r_{2}\right)\right) \cap . . f_{n}{ }^{-1}\left(S_{\epsilon}\left(r_{n}\right)\right) \cap\left(T_{s_{1}} f_{1}\right)^{-1}\left(S_{\epsilon}\left(r_{1}\right)\right) \cap \ldots \cap$ $\left(T_{s_{n}} f_{n}\right)^{-1}\left(S_{\epsilon}\left(r_{n}\right)\right)$ for $r_{1}, \ldots, r_{n} \in C$, where $S_{\epsilon}\left(r_{k}\right)=\left\{x \in C|| x-r_{k} \mid<\epsilon\right\}(k=1, \ldots, n)$. If some $L\left(r_{1}, \ldots, r_{n}\right)$ is $F, S$-thick, then it suffices for the $Y$ of $(h)$ with $r_{1}=c_{1}, \ldots, r_{n}=c_{n}$. Assume that no $L\left(r_{1}, \ldots, r_{n}\right)$ is F,S-thick. A contradiction shall be deduced. For each non-empty $L\left(r_{1}, \ldots, r_{n}\right)$ and for each $\lambda \in \operatorname{MM}(F), \exists s \in S, \exists g \in Z\left(L\left(r_{1}, \ldots, r_{n}\right)\right)$ such that $\lambda\left(T_{s}(g)\right) \neq 0$ by $(k)$ of Theorem 4.3. In particular, if $\lambda$ is invariant, then $\lambda(\mathrm{g})=\lambda\left(\mathrm{T}_{\mathrm{s}}(\mathrm{g})\right) \neq 0$. Let $\left\langle\mathrm{e}\left(\mathrm{x}_{\mathrm{v}}\right)\right\rangle$ he a net in $\mathrm{MM}(\mathrm{F})$ such that

$\lambda=w *-\lim _{v} e\left(x_{v}\right)$. Then for $i=1, \ldots, n, \exists N_{1}$ such that $v \geq N_{1}-\left|f_{1}\left(x_{v}\right)-\lambda f_{i}\right|<\epsilon$ and

$\left|\mathrm{T}_{\mathrm{s}_{1}} \mathrm{f}_{1}\left(\mathrm{x}_{\mathrm{v}}\right)-\lambda \mathrm{f}_{\mathrm{i}}\right|<\epsilon$; this entails that $v \geq \mathrm{N}_{1}, \mathrm{~N}_{2}, \ldots . \mathrm{N}_{\mathrm{n}}-\left|\mathrm{f}_{1}\left(\mathrm{x}_{\mathrm{v}}\right)-\lambda \mathrm{f}_{1}\right|<\epsilon$ and $\left|T_{s_{i}} f_{1}\left(x_{v}\right)-\lambda f_{i}\right|<\epsilon(i=l, \ldots, n)-x_{v} \in L\left(\lambda f_{1}, \ldots, \lambda f_{n}\right)$. For $L\left(\lambda f_{1}, \ldots, \lambda f_{n}\right), \exists g \in Z\left(L\left(\lambda f_{1}, \ldots, \lambda f_{n}\right)\right)$ with $\lambda(g) * 0$, as previously noted, so $g\left(x_{v}\right)=0$ for all $v \geq N_{1}, N_{2}, \ldots, N_{n}$. Therefore,

$\lambda(g)=\lim _{v} e\left(x_{v}\right) g=0$, a contradiction.

$d \Rightarrow e, e \rightarrow d, f-g, h \rightarrow e:$ Easy

QED

\section{REFERENCES}

1. Berglund, John F., Junghenn, Hugo D., and Milnes, Paul. Compact Right Topological Semigroups and Generalizations of Almost Periodicity. Lecture Notes in Mathematics No. 663. Berlin: Springer-Verlag, 1978.

2. Junghenn, Hugo D. "Amenability of Function Spaces on Thick Sub-semigroups." Proceedings of the American Mathematical Society, Vol. 75, No. 1 (1979), 37-41.

3. Lau, Anthony To-Ming. "Extremely Amenable Algebras." Pacific Joumal of Mathematics, Vol. 33, No. 2 (1970), 329-336.

4. — "Action of topological semigroups, invariant means, and fixed points." Studia Mathematica, Vol. 43 (1972), 139-156.

5. Mitchell, Theodore. "Constant Functions and Left Invariant Means on Semigroups." Transactions of the American Mathematical Society, Vol. 119 (1965 August). 244-261. 
6. Sakai, Kokichi. "On amenable transformation semigroups."

I. Journal of Mathematics, Kyoto Univ. 16-3 (1976), 555-595.

II. Ibid., 597-626.

III. Scientific Reports, Kagoshima Univ., No. 25 (1976 September), 31-51.

7. Simmons, George F. Introduction to Topology and Modern Analysis. New York: McGrawHill Book Co., 1963.

8. Wilde, Carroll, and Witz. Klaus. "Invariant Means and the Stone-Cech Compactification." Pacific Journal of Mathematics, Vol. 21 (1967), 577-586. 


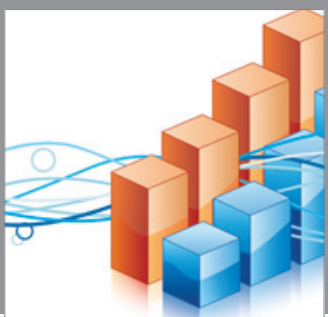

Advances in

Operations Research

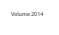

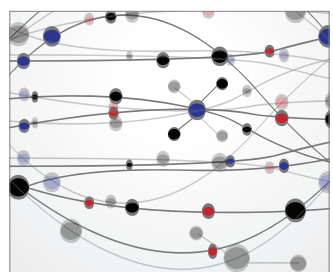

\section{The Scientific} World Journal
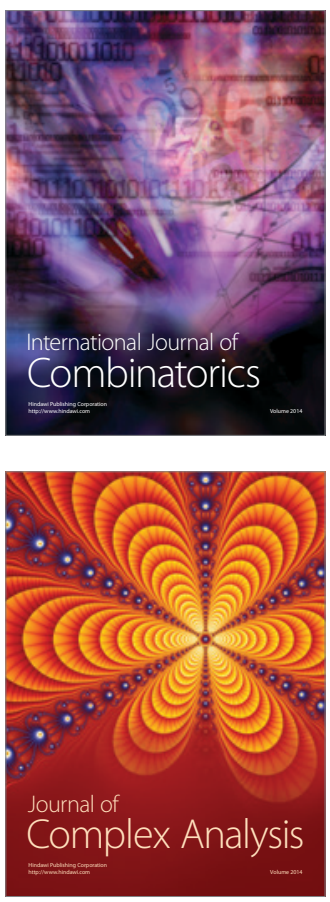

International Journal of

Mathematics and

Mathematical

Sciences
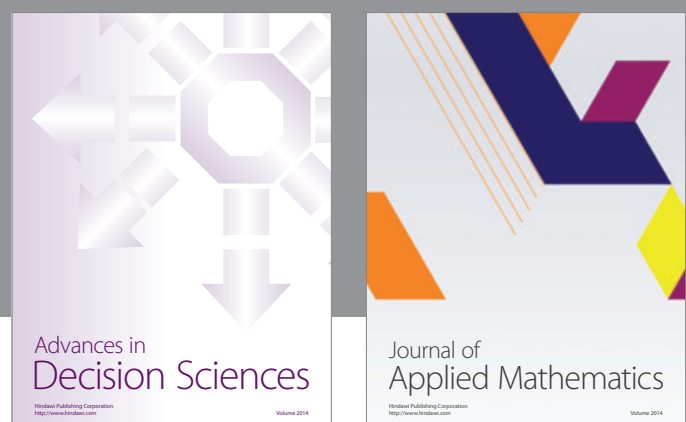

Journal of

Applied Mathematics
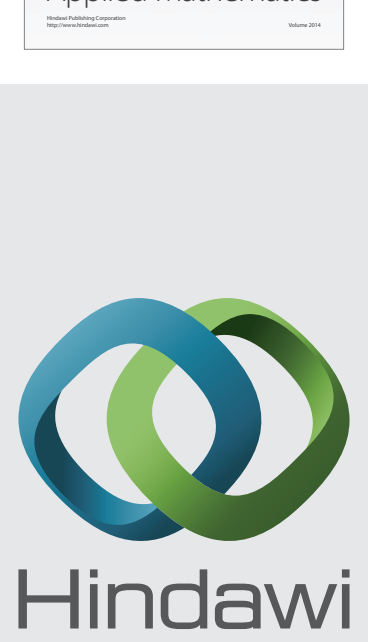

Submit your manuscripts at http://www.hindawi.com
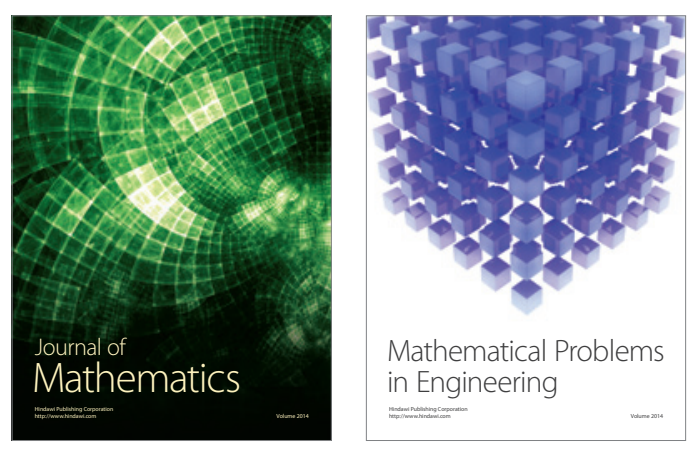

Mathematical Problems in Engineering
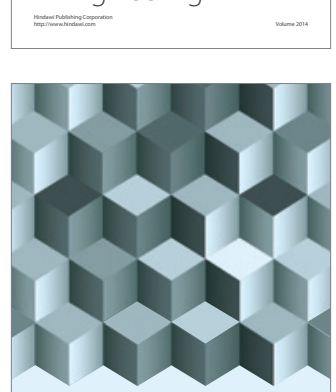

Journal of

Function Spaces
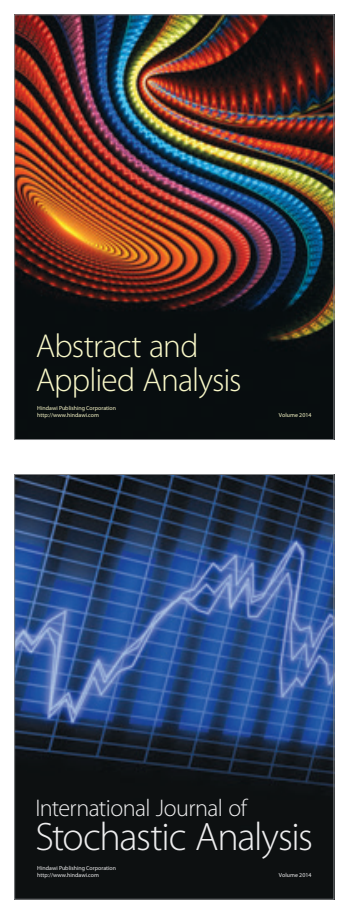

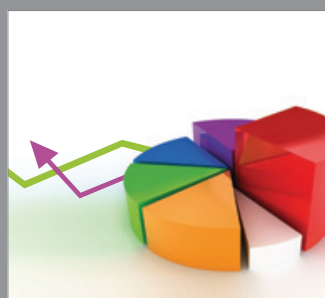

ournal of

Probability and Statistics

Promensencen
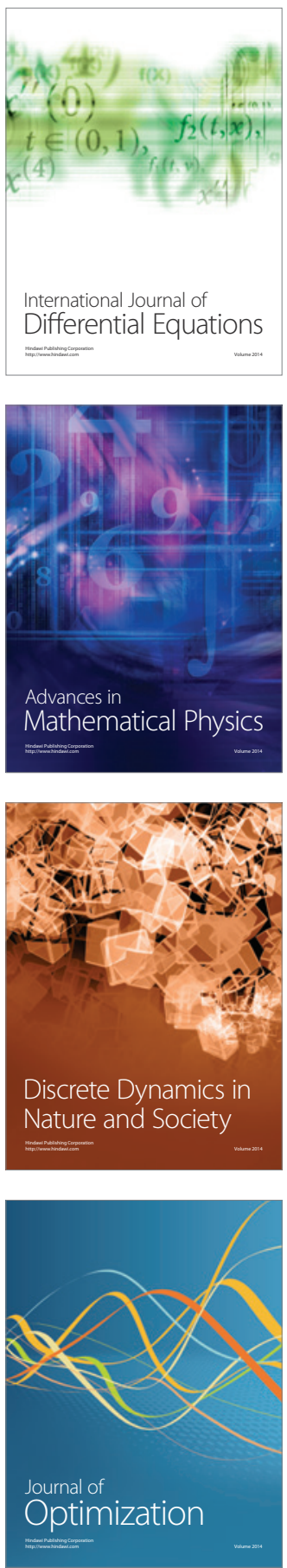